\section{Persistência da hanseníase em redes de convívio domiciliar: sobreposição de casos e vulnerabilidade em regiões endêmicas no Brasil}

\author{
Persistence of leprosy in household social \\ networks: overlapping cases and vulnerability \\ in endemic regions in Brazil
}

\section{Persistencia de la hanseniasis en redes de convivencia domiciliaria: sobreposición de casos y vulnerabilidad en regiones endémicas en Brasil}

Reagan Nzundu Boigny 1

Eliana Amorim de Souza 2

Helizandra Simoneti Bianchini Romanholo 3,4

Olivia Dias de Araújo 5

Telma Maria Evangelista de Araújo 5

Maria Angélica Gomes Carneiro 1

Monique Dutra Fonseca Grijó 6

Nubia de Lourdes Ferreira Bastos Henz 7

Adriana da Silva dos Reis 1

Maria Solange Araújo Paiva Pinto 1

Jaqueline Caracas Barbosa 1

Alberto Novaes Ramos Jr. 1

doi: $10.1590 / 0102-311 \times 00105318$

\section{Resumo}

O estudo tem como objetivo analisar a magnitude da ocorrência e os perfis sociodemográfico, econômico e clínico de casos de hanseníase vinculados à redes de convívio domiciliar (RCD) com sobreposição da doença em municípios dos estados da Bahia, do Piauí e de Rondônia, Brasil, no período de 2001 a 2014. Trata-se de estudo transversal, com dados primários e secundários de casos novos de hanseníase, notificados no Sistema de Informação de Agravos de Notificação (SINAN) e residentes nos municípios. Foram realizadas a aplicação de instrumento padronizado aos casos novos e a revisão de dados em prontuários e na base do SINAN. De um total de $1.032(29,6 \%)$ casos de hanseníase abordados, $538(52,1 \%)$ tinham mais de um caso em sua RCD. Maior frequência de pessoas do sexo feminino (292; 54,3\%), com idade entre 41 a 60 anos (240; 44,6\%), ensino fundamental (272; 50,6\%), renda menor que um salário mínimo (265; 49,3\%) e residindo com cinco pessoas ou mais (265; 49,3\%). A ocorrência de sobreposição de casos na RCD foi associada, na análise multivariada, a residir em municípios do Estado de Rondônia ( $R P=1,23$; IC95\%: 1,07-1,43; $p=0,003)$, assim como morar com três a quatro pessoas no mesmo domicílio $(R P=1,66$; IC95\%: 1,11-2,49; $p=0,014)$ e ter reação hansênica $(R P=1,31 ;$ IC95\%: 0,99-1,70; $p=0,050)$. A repetição de casos de hanseníase em uma mesma $R C D$ representa um evento frequente nos cenários abordados. Sua ocorrência deve ser considerada como indicador sentinela de maior gravidade epidemiológica para a vigilância na rede de atenção básica à saúde. Ressalta-se o caráter de vulnerabilidade das famílias acometidas.

Hanseníase; Transmissão de Doença Infecciosa; Monitoramento Epidemiológico

\section{Correspondência}

E. A. Souza

Instituto Multidisciplinar em Saúde, Universidade Federal da Bahia.

Rua Hormindo de Barros 58, Vitória da Conquista, BA 45029-094, Brasil.

amorim_eliana@yahoo.com.br

1 Faculdade de Medicina, Universidade Federal do Ceará, Fortaleza, Brasil.

2 Instituto Multidisciplinar em Saúde, Universidade Federal da Bahia, Vitória da Conquista, Brasil.

3 Faculdade de Ciências Biomédicas de Cacoal, Cacoal, Brasil.

4 Secretaria Municipal de Saúde de Cacoal, Cacoal, Brasil.

5 Universidade Federal do Piaui, Teresina, Brasil.

6 Centro de Referência Municipal para Hanseníase, Secretaria Municipal de Saúde, Vitória da Conquista, Brasil.

7 Secretaria Estadual de Saúde de Rolim de Moura, Rolim de Moura, Brasil. 


\section{Introdução}

A existência de clusters de casos novos de hanseníase envolvendo diferentes estados do Brasil revela a manutenção de elevada carga da doença em áreas delimitadas 1,2. Em 2017, com coeficiente de detecção geral de 12,94 casos por 100 mil habitantes, o país permaneceu dentro de padrões considerados de alta endemicidade 2,3. Nesse cenário, destacam-se as regiões Centro-oeste, Norte e Nordeste, as quais apresentaram, no ano de 2017, coeficientes de detecção de, respectivamente, 33,84, 28,82 e 20,58 casos novos por 100 mil habitantes, com maior expressão principalmente em populações com diferentes dimensões de vulnerabilidade 4,5 . De fato, muitas comunidades têm permanecido sob risco de adoecer, desenvolver incapacidade física e sofrer estigma associado à hanseníase, mantendo e/ou ampliando o ciclo da pobreza, elementos típicos de doenças tropicais negligenciadas 4,5,6.

A Organização Mundial da Saúde (OMS) define como estratégia principal para o controle da hanseníase o diagnóstico precoce associado ao tratamento oportuno de todos os casos 7 . Nesse sentido, ao reconhecer o risco acrescido em contatos de casos de hanseníase de infecção por Mycobacterium leprae e adoecimento, reforça-se que as ações de vigilância devem ser priorizadas nessa população, ocorrendo de forma contínua e sistemática 3,7,8,9,10. O Ministério da Saúde do Brasil definia contato, até 2016, como qualquer pessoa que residia ou tinha residido com o caso novo de hanseníase no âmbito do domicílio nos últimos cinco anos anteriores ao diagnóstico da doença ${ }^{8}$. Porém, ao verificar maior risco associado também a contatos fora do espaço domiciliar, o país incorporou, a partir de 2016, em suas diretrizes, a ampliação das ações de vigilância para essa população. Definiu-se contato social como qualquer pessoa que conviva ou tenha convivido em relações familiares ou não de forma próxima e prolongada com o caso não tratado. Assim, todos os contatos domiciliares e sociais necessitariam ser acompanhados, submetidos a exame dermatoneurológico e ser alvo de ações de educação em saúde. Para os contatos, insere-se ainda a imunoprofilaxia como intervenção 3,11.

Considerando o longo período de incubação da hanseníase, é essencial que o seguimento dos contatos aconteça durante, no mínimo, cinco anos ${ }^{3}$. No Brasil, uma estratégia efetiva direcionada para a saúde da família e comunidades persiste como grande desafio para o cuidado longitudinal junto a redes de convívio domiciliar (RCD) acometidas pela hanseníase 12,13. Tais dificuldades poderão ser evidenciadas pela ocorrência de sobreposição de casos nesses contextos, inclusive envolvendo diferentes gerações 14,15 .

Alguns indicadores operacionais propostos pelo Ministério da Saúde confirmam falhas significativas dos serviços de saúde, a exemplo da proporção de contatos examinados entre os registrados nas coortes de 2017, em que o Brasil alcançou apenas 78,9\% de cobertura 2. Além disso, destaca-se o fato de que esse indicador não capta os contatos sociais, não verifica o seguimento dos contatos ao longo de cinco anos e, adicionalmente, não permite avaliar a qualidade das ações previstas, como o exame dermatoneurológico 3. De forma complementar, $81,8 \%$ dos casos foram curados nas coortes desse mesmo ano, reforçando a ideia de que são mantidas fontes de infecção nas comunidades, mesmo quando o diagnóstico é estabelecido 2,3.

Para além das falhas dos serviços de saúde que ampliam a vulnerabilidade programática diretamente relacionada à manutenção da hanseníase, outros determinantes sociais do processo saúde-doença atuam fortemente na sua persistência, a exemplo das más condições de educação, renda e moradia, dentre outras 5,15. Desse modo, ainda que se reconheça a importância de melhor compreender diferentes aspectos da vulnerabilidade social, a literatura pouco explora essa dimensão, em particular, em contextos marcados pela ocorrência de diferentes casos de hanseníase em uma mesma RCD. Alcançar essas perspectivas poderá subsidiar ações mais abrangentes que promovam a redução das iniquidades sociais e de saúde, assim como instituir novos indicadores relacionados à vigilância dos contatos, potencializando o controle da hanseníase no território de atuação das equipes de saúde da família 14.

Dessa forma, o presente estudo tem como objetivo analisar a magnitude da ocorrência e os perfis sociodemográfico, econômico e clínico de casos de hanseníase vinculados à RCD com sobreposição da doença em municípios dos estados da Bahia, do Piauí e de Rondônia no período de 2001 a 2014. 


\section{Métodos}

\section{Desenho e população do estudo}

Trata-se de um estudo transversal com casos referência de hanseníase que tenham relatado, pelo menos, um caso adicional da doença em sua RCD. Neste estudo, casos referência foi considerado como o primeiro caso de hanseníase notificado no período em análise e que faz parte de uma RCD. A RCD inclui, além do caso referência de hanseníase, todos os contatos domiciliares e sociais, conforme definição do Ministério da Saúde 3, assim como os contatos domiciliares e sociais que se tornaram casos de hanseníase (casos coprevalentes). O reconhecimento de, no mínimo, dois casos de hanseníase em uma RCD foi definido como "sobreposição" da doença.

A identificação das RCD foi realizada a partir de dados primários de casos novos de hanseníase abordados nas pesquisas multicêntricas nacionais INTEGRAHANS Norte-Nordeste 16 e INTEGRAHANS-Piauí, coordenadas, respectivamente, pela Universidade Federal do Ceará (UFC) e pela Universidade Federal do Piauí (UFPI). Esses casos foram notificados no Sistema de Informação de Agravos de Notificação (SINAN), no período de 2001 a 2014, em municípios dos estados da Bahia e Piauí e, de 2001 a 2012, em municípios do Estado de Rondônia.

\section{Local do estudo}

O estudo abrangeu estados das regiões Norte e Nordeste, os quais fazem parte de importantes clusters de risco para a ocorrência de hanseníase no Brasil 1,2. Na Bahia, foram inseridos os municípios de Vitória da Conquista e Tremedal; no Piauí, o Município de Floriano; e, em Rondônia, os municípios de Cacoal e Rolim de Moura (Figura 1). Vitória da Conquista é o único município de porte médio do estudo, com estimativa populacional, para 2017, de 348.718 habitantes. Tremedal, Floriano, Cacoal e Rolim de Moura são de pequeno porte, com população de 17.700; 58.969; 88.507 e 54.702 habitantes, respectivamente (Instituto de Pesquisa Econômica Aplicada. http://atlasbrasil.org.br/2013/pt/per fil_uf/bahia, acessado em 25/Set/018; Instituto Brasileiro de Geografia e Estatística. https://cidades. ibge.gov.br/, acessado em 25/Set/2018).

O Município de Tremedal tem mais da metade da sua população residindo na zona rural e apresenta o maior percentual de pessoas em situação de extrema pobreza (24,3\%) entre os municípios do estudo. O índice de Gini, que revela situações de desigualdade, foi de 0,55; 0,48; 0,55; 0,57 e 0,53, respectivamente, para Vitória da Conquista, Tremedal, Floriano, Cacoal e Rolim de Moura (Instituto de Pesquisa Econômica Aplicada. http://atlasbrasil.org.br/2013/pt/perfil_uf/bahia, acessado em 25/Set/018; Instituto Brasileiro de Geografia e Estatística. https://cidades.ibge.gov.br/, acessado em 25/Set/2018).

Em todos os municípios, a atenção primária à saúde está organizada a partir da Estratégia Saúde da Família (ESF), com coberturas populacionais variando de 48,1\% (Vitória da Conquista) a 100\% (Floriano, Tremedal, Cacoal e Rolim de Moura). Somente o Município de Tremedal não dispõe de serviço de atenção especializado para hanseníase (Ministério da Saúde. Histórico de cobertura da saúde da família. http://dab.saude.gov.br/portaldab/historico_cobertura_sf.php, acessado em 25/Set/2018).

\section{Fonte e coleta de dados}

Para abordagem dos casos novos de hanseníase em seu domicílio, foi realizada consulta de campos relacionados ao endereço de residência na base de dados do SINAN-hanseníase. Para todos os casos localizados nas áreas de abrangência das unidades de atenção primária à saúde e que aceitaram participar do estudo ( $n=1.032$ casos novos), realizou-se a aplicação de instrumento estruturado. Considerando a possibilidade de viés de memória e/ou de desconhecimento sobre informações clínicas da época do diagnóstico e tratamento da hanseníase, foram realizadas consultas em prontuários, além da própria base do SINAN.

As variáveis explicativas (sociodemográficas, econômicas e clínicas) deste estudo incluíam: sexo, faixa etária, raça/cor, escolaridade, renda, recebimento de recursos do Programa Bolsa Família (PBF), 


\section{Figura 1}

Localização geográfica dos municípios participantes do estudo: Cacoal e Rolim de Moura (Estado de Rondônia); Vitória da Conquista e Tremedal (Estado da Bahia); e Floriano (Estado do Piauí), Brasil.

1a) Rondônia

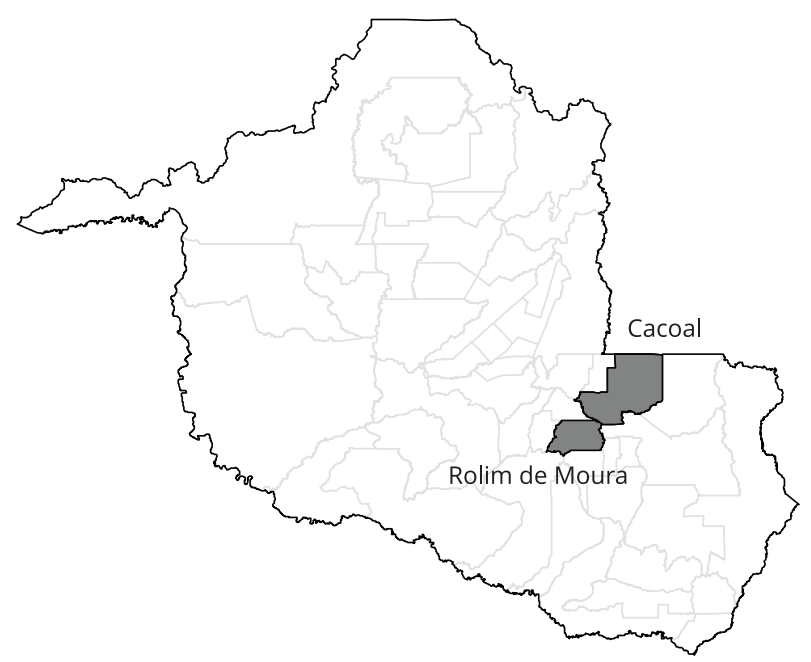

1c) Piauí

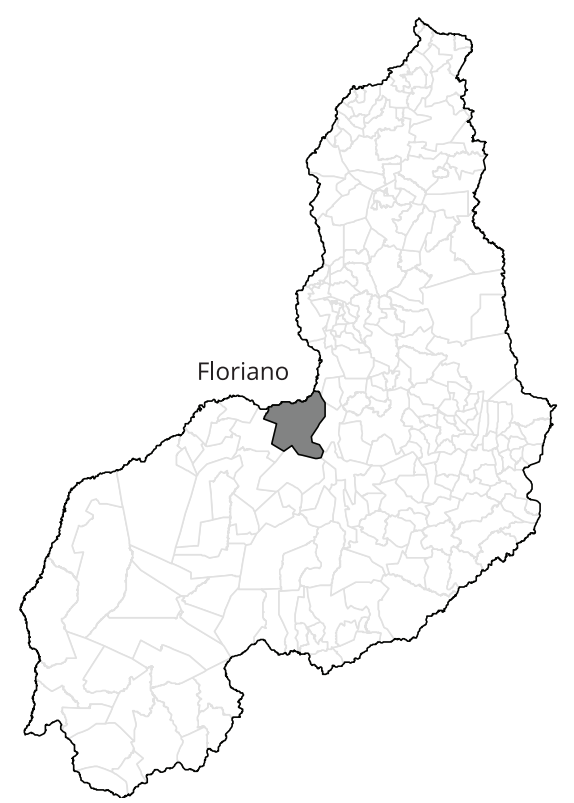

1b) Bahia
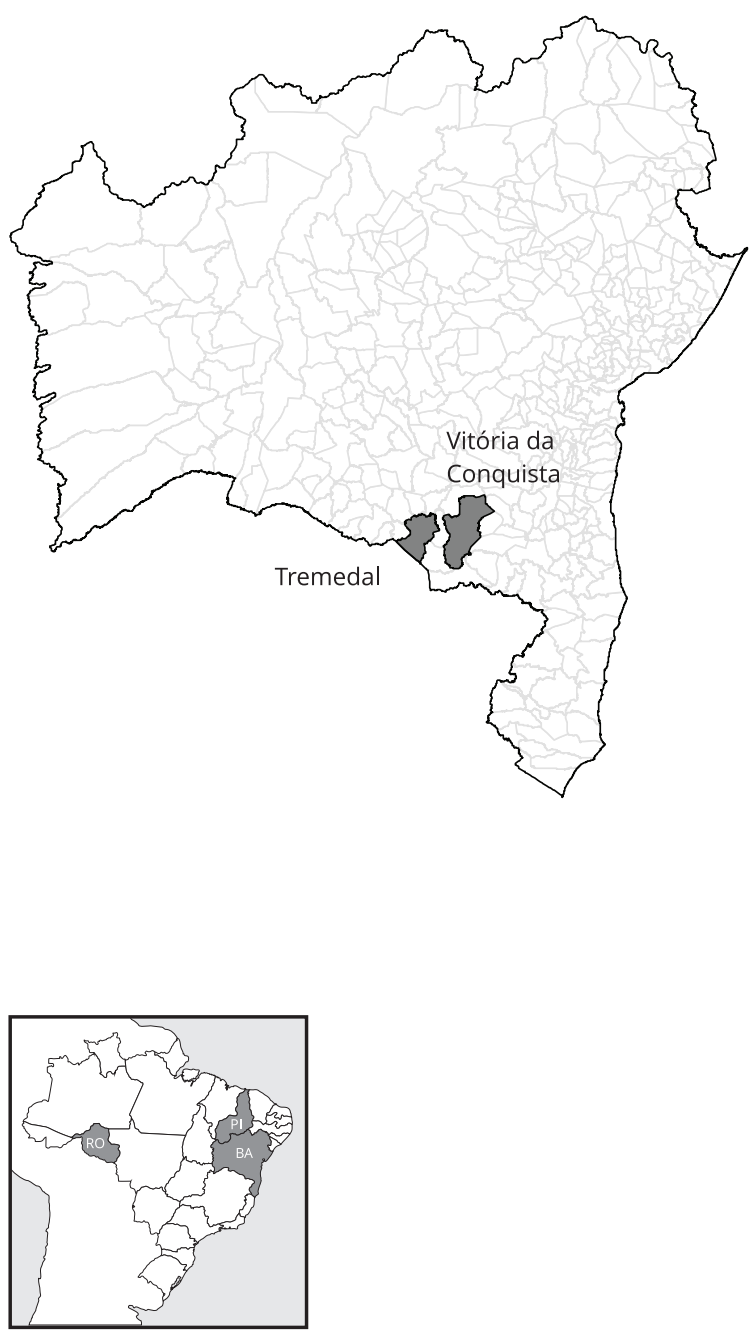

número de pessoas por residência, Estado de residência, forma clínica da doença, classificação operacional, reação hansênica e presença de outros casos de hanseníase anterior e/ou posteriormente ao seu diagnóstico entre contatos domiciliares e sociais (neste estudo, designado como caso coprevalente). A variável renda foi recategorizada considerando o salário mínimo de $\mathrm{R} \$ 724,00$ no momento da pesquisa. Foi definida como variável desfecho a "sobreposição" a fim de revelar a existência de RCD com a presença de mais de um caso da doença. 


\section{Consolidação e análise de dados}

Para entrada e análise dos dados, foram utilizados os softwares Epi Info 3.5.1 (https://www.cdc.gov/ epiinfo/index.html) e Stata 11.2 (https://www.stata.com). Para analisar a associação entre as variáveis explicativas e o desfecho, foram calculadas as razões de prevalência (RP) com os seus respectivos intervalos de 95\% de confiança (IC95\%). A significância estatística foi fixada em p < 0,05 e indicou as variáveis que seguiram para a sequência de análise.

Para a análise multivariada, foram utilizados procedimentos de regressão logística cuja variável dependente foi categorizada pela recorrência de sobreposição de casos nas RCD (binária, sim/não). O nível de significância para inclusão de variáveis foi estabelecido em $5 \%$, utilizado para qualquer uma das categorias da variável-resposta, avaliando questões de interação e confusão. Procederam-se às estimativas de odds ratio (OR) brutos e ajustados e seus respectivos IC95\% para aquelas variáveis que permaneceram no modelo explicativo.

\section{Aspectos éticos}

O projeto foi submetido e aprovado pelo Comitê de Ética em Pesquisa (CEP) da UFC, sob o número 544.962, de 28 de fevereiro de 2014, e da UFPI, sob o número 1.115.818, de 17 de julho de 2015.

\section{Resultados}

Dos 3.483 casos novos de hanseníase notificados nos municípios de 2001 a 2014, 1.032 (29,6\%) foram abordados em seus domicílios, localizados em territórios cobertos pela atenção primária à saúde. Desse total, 29,7\% (307/1.032) residiam no Estado da Bahia; 28,3\% (292/1.032), no Piauí; e 41,9\% (433/1.032), em Rondônia. A maioria ( $n=538 ; 52,1 \%)$ afirmou ter sobreposição de casos de hanseníase na mesma RCD, reforçando a ocorrência da doença em clusters também em uma perspectiva familiar e social (Tabela 1).

Houve maior proporção de sobreposição de casos quando o caso referência era do sexo feminino ( $\mathrm{n}=292 ; 54,3 \%)$, à exceção dos municípios da Bahia $(\mathrm{n}=68 ; 47,9 \%)$. A faixa etária de maior ocorrência foi aquela entre 41 a 60 anos para os municípios da Bahia $(\mathrm{n}=71 ; 50,7 \%)$ e de Rondônia $(\mathrm{n}=122$; 49,2\%), e entre 15 a 40 anos para o município do Piauí ( $n=54 ; 36,5 \%)$. No geral, a raça/cor parda foi a mais frequente $(\mathrm{n}=265 ; 49,3 \%)$, do mesmo modo que ter cursado até o Ensino Fundamental $(\mathrm{n}=$ 272; 50,6\%) (Tabela 1).

Receber menos de um salário mínimo foi uma condição presente em 49,3\% ( $n=265)$ dos casos de sobreposição, estando os municípios da Bahia com os mais altos percentuais ( $\mathrm{n}=103 ; 72,4 \%$ ). Por outro lado, nos municípios de Rondônia, foram identificados os maiores percentuais de pessoas que recebiam acima de dois salários mínimos ( $\mathrm{n}=38$; 15,3\%). Do mesmo modo, nesse estado, são menores os percentuais de pessoas que recebem recursos do PBF: $14,1 \%(n=35)$. Em todos os municípios, 21,6\% (116) relataram ter esse benefício. Residir em domicílios com cinco ou mais pessoas representou um contexto mais frequente $(n=265 ; 49,3 \%)$. A Bahia apresentou os maiores percentuais $(n=80$; 56,3\%), enquanto o Piauí, os menores ( $\mathrm{n}=57 ; 38,5 \%$ ) nessa condição (Tabela 1 ).

Constatou ser maior a proporção de casos classificados com a forma clínica dimorfa ( $\mathrm{n}=181$; 33,6\%) nas RCD com sobreposição da doença, à exceção do Município do Piauí, em que a forma indeterminada foi verificada em $40,5 \%$ dos casos $(n=60)$, principalmente na classificação operacional paucibacilar ( $\mathrm{n}=87 ; 58,8 \%$ ). Já, na Bahia, 66,9\% ( $\mathrm{n}=95)$ foram classificados como multibacilares. Identificou-se a ocorrência de episódios reacionais em 39,2\% ( $\mathrm{n}=211)$ dos casos, com destaque para os municípios de Rondônia ( $\mathrm{n}=113 ; 45,6 \%)$ e da Bahia ( $\mathrm{n}=57 ; 40,1 \%$ ) (Tabela 1$)$.

A Tabela 2 demonstra que os municípios do Estado de Rondônia, com 57,3\% (248/433) de sobreposição de casos, apresentaram RP de 1,23 (IC95\%: 1,07-1,43; p = 0,003) em relação ao Estado da Bahia, mas sem diferença significativa em relação a sexo, raça/cor, renda e ter PBF. No entanto, casos referência com idade acima de 60 anos tiveram menos probabilidade de ter sobreposição de caso $(\mathrm{RP}=$ 0,71; IC95\%: 0,60-0,83; $\mathrm{p}<0,001)$ quando comparado com indivíduos da faixa etária de 15 a 40 anos. Por outro lado, ter ensino médio ( $R P=1,25$; IC95\%: 1,05-1,40; $p=0,001)$ e residir com três 
Tabela 1

Caracterização demográfica, socioeconômica e clínica da sobreposição de casos de hanseníase em redes de convívio domiciliar (RCD), por local de estudo. Estados de Rondônia, Bahia e Piauí, Brasil, 2001 a 2014.

\begin{tabular}{|c|c|c|c|c|c|c|c|c|}
\hline \multirow[t]{2}{*}{ Variáveis } & \multicolumn{2}{|c|}{ Bahia } & \multicolumn{2}{|c|}{ Piauí } & \multicolumn{2}{|c|}{ Rondônia } & \multicolumn{2}{|c|}{ Geral } \\
\hline & $\mathbf{n}$ & $\%$ & $\mathbf{n}$ & $\%$ & $\mathbf{n}$ & $\%$ & $\mathrm{n}$ & $\%$ \\
\hline \multicolumn{9}{|l|}{ Demográficas } \\
\hline \multicolumn{9}{|l|}{ Sexo } \\
\hline Masculino & 74 & 52,1 & 73 & 49,3 & 99 & 39,9 & 246 & 45,7 \\
\hline Feminino & 68 & 47,9 & 75 & 50,7 & 149 & 60,1 & 292 & 54,3 \\
\hline \multicolumn{9}{|l|}{ Faixa etária (anos) } \\
\hline $0-14$ & 1 & 0,7 & 2 & 1,4 & 3 & 1,2 & 6 & 1,1 \\
\hline $15-40$ & 38 & 26,8 & 54 & 36,5 & 71 & 28,6 & 163 & 30,3 \\
\hline $41-60$ & 71 & 50,0 & 47 & 31,8 & 122 & 49,2 & 240 & 44,6 \\
\hline Acima de 60 & 32 & 22,5 & 45 & 30,3 & 52 & 21,0 & 129 & 24,0 \\
\hline \multicolumn{9}{|l|}{ Raça/Cor * } \\
\hline Branca & 25 & 17,6 & 17 & 11,5 & 76 & 30,7 & 118 & 21,9 \\
\hline Parda & 72 & 50,7 & 58 & 39,2 & 135 & 54,4 & 265 & 49,3 \\
\hline Negra & 38 & 26,8 & 44 & 29,7 & 30 & 12,1 & 112 & 20,8 \\
\hline \multicolumn{9}{|l|}{ Socioeconômicas } \\
\hline \multicolumn{9}{|l|}{ Escolaridade } \\
\hline Não estudou & 49 & 34,5 & 25 & 16,9 & 28 & 11,3 & 102 & 19,0 \\
\hline Ensino Fundamental & 68 & 47,9 & 71 & 48,0 & 133 & 53,6 & 272 & 50,6 \\
\hline Ensino Médio & 22 & 15,5 & 41 & 27,7 & 69 & 27,8 & 132 & 24,4 \\
\hline Ensino Superior & 3 & 2,1 & 11 & 7,4 & 18 & 7,3 & 32 & 6,0 \\
\hline \multicolumn{9}{|c|}{ Renda (salários mínimos) * } \\
\hline Menor de 1 & 103 & 72,5 & 50 & 33,8 & 112 & 45,2 & 265 & 49,3 \\
\hline $1-2$ & 26 & 18,3 & 69 & 46,6 & 76 & 30,7 & 171 & 31,8 \\
\hline Acima de 2 & 12 & 8,5 & 11 & 7,4 & 38 & 13,3 & 61 & 11,3 \\
\hline \multicolumn{9}{|l|}{ Recebe Bolsa Família } \\
\hline Não & 94 & 66,2 & 115 & 77,7 & 213 & 85,9 & 422 & 78,4 \\
\hline Sim & 48 & 33,8 & 33 & 22,3 & 35 & 14,1 & 116 & 21,6 \\
\hline \multicolumn{9}{|l|}{ Número de residentes * } \\
\hline Até 2 & 11 & 7,8 & 32 & 21,6 & 16 & 6,5 & 59 & 10,9 \\
\hline $3-4$ & 49 & 34,5 & 58 & 39,2 & 100 & 40,3 & 207 & 38,5 \\
\hline 5 ou mais & 80 & 56,3 & 57 & 38,5 & 128 & 51,6 & 265 & 49,3 \\
\hline Indeterminado & 10 & 7,1 & 60 & 40,5 & 45 & 18,2 & 115 & 21,4 \\
\hline \multicolumn{9}{|l|}{ Clínicas } \\
\hline \multicolumn{9}{|l|}{ Forma clínica * } \\
\hline Tuberculoide & 31 & 21,8 & 26 & 17,6 & 41 & 16,5 & 98 & 39,6 \\
\hline Dimorfa & 53 & 37,3 & 41 & 27,7 & 87 & 35,1 & 181 & 33,6 \\
\hline Virchowiana & 34 & 23,9 & 14 & 9,5 & 23 & 9,3 & 71 & 13,2 \\
\hline \multicolumn{9}{|c|}{ Classificação operacional * } \\
\hline Paucibacilar & 38 & 26,8 & 87 & 58,8 & 86 & 34,7 & 211 & 39,2 \\
\hline Multibacilar & 95 & 66,9 & 59 & 39,9 & 109 & 43,9 & 263 & 48,9 \\
\hline \multicolumn{9}{|l|}{ Reação hansênica } \\
\hline Não & 85 & 59,9 & 107 & 72,3 & 135 & 54,4 & 327 & 60,8 \\
\hline Sim & 57 & 40,1 & 41 & 27,7 & 113 & 45,6 & 211 & 39,2 \\
\hline
\end{tabular}

* Valor ignorado: raça/cor: 41 (Bahia = 7, Piauí = 29, Rondônia = 7); renda: 41 (Bahia = 1, Piauí = 18 e Rondônia = 22); número de residentes: 7 (Bahia = 2, Piauí = 1 e Rondônia = 4); forma clínica: 73 (Bahia = 14, Piauí = 7 e Rondônia = 52); classificação operacional: 64 (Bahia = 9, Piauí $=2$ e Rondônia $=53$ ). 
Tabela 2

Análise da sobreposição de casos de hanseníase em redes de convívio domiciliar (RCD) segundo variáveis demográficas, socioeconômicas e clínicas por local de estudo. Estados de Bahia, Piauí e Rondônia, Brasil, 2001 a 2014.

\begin{tabular}{|c|c|c|c|c|c|c|}
\hline Variáveis & $\begin{array}{l}\text { Total } \\
\text { n (\%) }\end{array}$ & $\begin{array}{l}\text { Não } \\
\text { n (\%) }\end{array}$ & $\begin{array}{c}\text { Sim } \\
\text { n (\%) }\end{array}$ & $\mathbf{R P}$ & IC95\% & Valor de $p$ \\
\hline \multicolumn{7}{|l|}{ Estados } \\
\hline Bahia & $307(29,7)$ & $165(53,7)$ & $142(46,3)$ & 1,00 & - & - \\
\hline Piauí & $292(28,3)$ & $144(49,3)$ & $148(50,7)$ & 1,09 & $0,92-1,29$ & 0,278 \\
\hline Rondônia & $433(42,0)$ & $185(42,7)$ & $248(57,3)$ & 1,23 & $1,07-1,43$ & 0,003 \\
\hline \multicolumn{7}{|l|}{ Demográficas } \\
\hline \multicolumn{7}{|l|}{ Sexo } \\
\hline Masculino & $465(45,1)$ & $219(47,1)$ & $246(52,9)$ & 1,00 & - & - \\
\hline Feminino & $567(54,9)$ & $275(58,5)$ & $292(51,5)$ & 0,97 & $0,86-1,09$ & 0,653 \\
\hline \multicolumn{7}{|l|}{ Faixa etária (anos) } \\
\hline $0-14$ & $15(1,5)$ & $9(60,0)$ & $6(40,0)$ & 0,66 & $0,35-1,24$ & 0,122 \\
\hline $15-40$ & $271(26,3)$ & $108(39,9)$ & $163(60,1)$ & 1,00 & - & - \\
\hline $41-60$ & $444(43,0)$ & $204(46,0)$ & $240(54,0)$ & 0,89 & $0,78-1,02$ & 0,111 \\
\hline Acima de 60 & $302(29,3)$ & $173(57,3)$ & $129(42,7)$ & 0,71 & $0,60-0,83$ & $<0,001$ \\
\hline \multicolumn{7}{|l|}{ Raça/Cor * } \\
\hline Branca & $224(23,5)$ & $106(47,3)$ & $118(52,7)$ & 1,00 & - & - \\
\hline Parda & $521(54,6)$ & $256(49,1)$ & $265(50,9)$ & 0,96 & $0,83-1,12$ & 0,649 \\
\hline Negra & $209(21,9)$ & $97(46,4)$ & $112(53,6)$ & 1,01 & $0,85-1,21$ & 0,849 \\
\hline \multicolumn{7}{|l|}{ Socioeconômicas } \\
\hline \multicolumn{7}{|l|}{ Escolaridade } \\
\hline Não estudou & $213(20,6)$ & $102(47,9)$ & $111(52,1)$ & 1,00 & - & - \\
\hline Ensino Fundamental & $543(52,6)$ & $272(50,1)$ & $271(49,9)$ & 1,04 & $0,88-1,23$ & 0,585 \\
\hline Ensino Médio & $219(21,2)$ & $132(60,3)$ & $87(39,7)$ & 1,25 & $1,05-1,50$ & 0,001 \\
\hline Ensino Superior & $57(5,5)$ & $32(56,1)$ & $25(43,9)$ & 1,17 & $0,89-1,53$ & 0,268 \\
\hline \multicolumn{7}{|l|}{ Renda (salários mínimos) * } \\
\hline Menos 1 & $506(53,3)$ & $241(47,6)$ & $265(52,4)$ & 0,99 & $0,82-1,21$ & 0,966 \\
\hline $1-2$ & $327(34,5)$ & $156(47,7)$ & $171(52,3)$ & 0,99 & $0,81-1,21$ & 0,956 \\
\hline Acima de 2 & $116(12,2)$ & $55(47,4)$ & $61(52,6)$ & 1,00 & - & - \\
\hline \multicolumn{7}{|l|}{ Recebe Bolsa Família } \\
\hline Não & $832(80,6)$ & $410(49,3)$ & $422(50,7)$ & 1,00 & - & - \\
\hline Sim & $200(19,4)$ & $84(42,0)$ & $116(58,0)$ & 1,14 & $0,99-1,30$ & 0,064 \\
\hline \multicolumn{7}{|l|}{ Número de residentes * } \\
\hline Até 2 & $141(13,9)$ & $82(58,2)$ & $59(41,8)$ & 1,00 & - & - \\
\hline $3-4$ & $375(37,0)$ & $168(44,8)$ & $207(55,2)$ & 1,31 & $1,06-1,63$ & 0,006 \\
\hline 5 ou mais & $498(49,1)$ & $233(46,8)$ & $265(53,2)$ & 1,27 & $1,02-1,57$ & 0,017 \\
\hline \multicolumn{7}{|l|}{ Clínicas } \\
\hline \multicolumn{7}{|l|}{ Forma clínica * } \\
\hline Indeterminada & $221(24,1)$ & $106(48,0)$ & $115(52,0)$ & 1,00 & & \\
\hline Tuberculoide & $212(23,2)$ & $114(53,8)$ & $98(46,2)$ & 0,89 & $0,73-1,08$ & 0,227 \\
\hline Dimorfa & $341(37,2)$ & $160(46,9)$ & $181(53,1)$ & 1,02 & $0,87-1,19$ & 0,809 \\
\hline Virchowiana & $142(15,5)$ & $71(50,0)$ & $71(50,0)$ & 0,96 & $0,78-1,18$ & 0,705 \\
\hline \multicolumn{7}{|c|}{ Classificação operacional * } \\
\hline Paucibacilar & $428(45,8)$ & $217(50,7)$ & $211(49,3)$ & 1,00 & & \\
\hline Multibacilar & $506(54,2)$ & $243(48,0)$ & $263(52,0)$ & 1,05 & $0,93-1,19$ & 0,415 \\
\hline \multicolumn{7}{|l|}{ Reação hansênica } \\
\hline Não & $662(64,1)$ & $335(50,6)$ & $327(49,4)$ & 1,00 & & \\
\hline Sim & $379(35,9)$ & $159(43,0)$ & $211(57,0)$ & 1,15 & $1,02-1,29$ & 0,019 \\
\hline
\end{tabular}

IC95\%: intervalo de 95\% de confiança; RP: razão de prevalência.

* Valor ignorado: raça/cor: 41 (Bahia = 7, Piauí = 29, Rondônia = 7); renda: 41 (Bahia = 1, Piauí = 18 e Rondônia = 22); número de residentes: 7 (Bahia = 2, Piauí = 1 e Rondônia = 4); forma clínica: 73 (Bahia = 14, Piauí = 7 e Rondônia = 52); classificação operacional: 64 (Bahia = 9, Piauí = 2 e Rondônia = 53). 
a quatro pessoas $(\mathrm{RP}=1,31$; IC95\%: $1,06-1,63 ; \mathrm{p}=0,006)$ ou acima de cinco pessoas na residência $(\mathrm{RP}=1,27$; IC95\%: 1,02-1,57; $\mathrm{p}<0,017)$ aumentaram a ocorrência da sobreposição.

Verificou-se a existência de maior percentual de sobreposição da hanseníase em casos da forma clínica dimorfa e com classificação multibacilar, porém sem significância estatística. No entanto, verificou-se associação significativa em casos com ocorrência de episódios de reação hansênica $(\mathrm{RP}=1,15$; IC95\%: 1,02-1,29; $\mathrm{p}=$ 0,019) (Tabela 2).

Na análise multivariada, a sobreposição de casos manteve-se associada ao fato de residir no Estado de Rondônia ( $\mathrm{RP}=1,42$; IC95\%: 1,05-1,92; $\mathrm{p}=0,024)$, morar com três a quatro pessoas no mesmo domicílio ( $R P=1,66$; IC95\%: 1,11-2,49; $p=0,014)$ e ter tido reação hansênica ( $R P=1,31$; IC95\%: 0,991,$70 ; p=0,005$ ) (Tabela 3). Estar na faixa etária acima de 60 anos ou mais ( $R P=0,54$; IC95\%: 0,38-0,75; $\mathrm{p}<0,001)$ apresentou menor associação com sobreposição.

\section{Discussão}

A sobreposição de casos de hanseníase foi verificada na população foco deste estudo, em contextos de alta endemicidade no país. Trata-se de uma abordagem inovadora na rede de atenção à saúde, trazendo possibilidades para ampliação da configuração de risco no espaço da família e de redes sociais. Avaliando todos os municípios dos três estados envolvidos, em mais da metade dos casos abordados, reconheceu-se, pelo menos, uma outra pessoa que teve a doença e que fazia parte da sua RCD, com destaque para os municípios do Estado de Rondônia. Configurou-se, ainda, na análise multivariada, associação do evento de sobreposição frente à ocorrência de episódio reacional hansênico.

\section{Tabela 3}

Análise multivariada da sobreposição de casos de hanseníase em redes de convívio domiciliar (RCD) por local de estudo. Estados de Bahia, Piauí e Rondônia, Brasil, 2001 a 2014.

\begin{tabular}{|c|c|c|c|c|c|c|}
\hline \multirow[t]{2}{*}{ Variáveis } & \multicolumn{3}{|c|}{ OR bruto } & \multicolumn{3}{|c|}{ OR ajustado } \\
\hline & OR & IC95\% & Valor de $p$ & OR & IC95\% & Valor de $p$ \\
\hline \multicolumn{7}{|l|}{ Estados } \\
\hline Bahia & 1,00 & & & 1,00 & & \\
\hline Piauí & 1,19 & $0,87-1,65$ & 0,278 & 1,28 & $0,91-1,79$ & 0,152 \\
\hline Rondônia & 1,56 & $1,16-2,09$ & 0,003 & 1,42 & $1,05-1,92$ & 0,024 \\
\hline \multicolumn{7}{|l|}{ Faixa etária (anos) } \\
\hline $0-14$ & 0,44 & $0,15-1,28$ & 0,131 & 0,32 & $0,09-1,10$ & 0,071 \\
\hline $15-40$ & 1,00 & & & 1,00 & & \\
\hline $41-60$ & 0,78 & $0,57-1,06$ & 0,111 & 0,79 & $0,58-1,07$ & 0,132 \\
\hline Acima de 60 & 0,49 & $0,35-0,69$ & $<0,001$ & 0,54 & $0,38-0,75$ & $<0,001$ \\
\hline \multicolumn{7}{|l|}{ Escolaridade } \\
\hline Não estudou & 1,00 & & & 1,00 & & \\
\hline Ensino Fundamental & 1,09 & $0,79-1,49$ & 0,586 & 0,95 & $0,66-1,37$ & 0,786 \\
\hline Ensino Médio & 1,65 & $1,13-2,42$ & 0,010 & 1,41 & $0,92-2,18$ & 0,117 \\
\hline Ensino Superior & 1,39 & $0,77-2,51$ & 0,269 & 0,98 & $0,52-1,85$ & 0,959 \\
\hline \multicolumn{7}{|l|}{ Número de residentes } \\
\hline Até 2 & 1,00 & & & 1,00 & & \\
\hline $3-4$ & 1,71 & $1,16-2,43$ & 0,007 & 1,66 & $1,11-2,49$ & 0,014 \\
\hline 5 ou mais & 1,58 & $1,08-2,31$ & 0,018 & 1,46 & $0,98-2,17$ & 0,061 \\
\hline \multicolumn{7}{|l|}{ Reação hansênica } \\
\hline Não & 1,00 & & & 1,00 & & \\
\hline Sim & 1,36 & $1,05-1,76$ & 0,019 & 1,31 & $0,99-1,70$ & 0,050 \\
\hline
\end{tabular}


Os altos percentuais de sobreposição encontrados neste estudo, juntamente com o fato de que quase $65 \%$ dos casos referência apresentaram diagnóstico posterior a outros casos próximos, reafirmam maior probabilidade de adoecimento entre pessoas da mesma RCD. Revelam ainda possíveis falhas no desenvolvimento de ações de vigilância do contato, com manutenção de focos de transmissão ativa da doença 3,7,9. A maior frequência e associação da sobreposição de casos nos municípios do Estado de Rondônia, quando comparados aos municípios da Bahia, pode ser explicada por serem espaços de maior endemicidade no país. Destaca-se que os municípios de Rolim de Moura (coeficiente de detecção de casos novos: 108,2 por 100 mil habitantes) e Cacoal (coeficiente de detecção de casos novos: 43,2 por 100 mil habitantes) estão entre os três municípios do Estado de Rondônia e do Brasil com maior ocorrência de casos novos 1,2 .

Outros estudos evidenciam a existência de mais de um caso em contextos familiares, a exemplo do realizado em Vitória, no Espírito Santo, onde 44,7\% dos casos novos diagnosticados informaram essa condição ${ }^{17}$. A identificação das mesmas cepas de $M$. leprae entre os familiares de casos de hanseníase reforça que, além dos contatos constituírem uma população de alto risco para infecção, podem atuar como fonte de infecção 9 , o que deveria ser o suficiente para efetivar uma vigilância cuidadosa e longitudinal das RCD.

O estudo da hanseníase em uma perspectiva além do indivíduo acometido deve considerar o reconhecimento de possíveis RCD como elemento importante para melhor compreensão da dinâmica de transmissão e da adoção de ações oportunas de vigilância e controle. Ressalta-se, portanto, a necessidade de ampliar o foco das ações para os casos entre os contatos fora do domicílio, em especial nas áreas de maior endemicidade 3,18,19,20. No entanto, os dados deste estudo sugerem que a experiência do adoecimento entre pessoas de uma mesma RCD parece não ser suficiente para desdobrar em ações de atenção e cuidado longitudinal, na perspectiva da integralidade do cuidado e da redução da carga da doença 21 .

Embora o estudo não tenha revelado significância estatística entre sobreposição de casos de hanseníase nas RCD e aspectos sociais e econômicos, verificou-se que quase 70\% dos casos ocorreram entre indivíduos com baixa escolaridade ou que nunca tinham estudado. De fato, essa situação também é comumente verificada em outros estudos 5,14. Do mesmo modo, aproximadamente, 50\% recebiam menos de um salário mínimo, e um percentual significativo de pessoas apresentava perfis social e econômico compatíveis para o cadastrado no PBF 4.

É preciso considerar que alguns desses municípios, foco do estudo, apresentam perfil social com pobreza e extrema pobreza. Entre eles, destaca-se o Município de Tremedal, localizado na região sudoeste da Bahia, uma vez que já foi considerado um dos piores municípios do país em termos de condições de vida. Nessa perspectiva, aspectos relacionados à vulnerabilidade social necessitam ser melhor compreendidos na manutenção da hanseníase, com vistas a contribuir para planejamento e execução de ações efetivas no cuidado das pessoas afetadas, mas também na vigilância de contatos, incluindo as RCD.

A pouca escolaridade, além de ser indicativo proxy de baixo poder aquisitivo, desdobra-se em maiores dificuldades para compreensão e incorporação de aspectos importantes relacionados a práticas de educação em saúde e autocuidado 20 , elementos essenciais para controle da doença 3,7. Do mesmo modo, receber até um salário mínimo pode ser uma condição relacionada a dificuldades de acesso a serviços de saúde e a outras questões relevantes para o controle da dinâmica de transmissão das doenças tropicais negligenciadas, para além da hanseníase 6 .

A relativa ausência de trabalhos publicados que discutam as RCD com sobreposição da hanseníase e suas condições socioeconômicas revelam a necessidade de ampliar os estudos nessa área. Entretanto, já se reconhece a associação da hanseníase com a pobreza nos territórios com maior vulnerabilidade 4,5,15,21, a exemplo do realizado no Estado do Pará, onde verificou-se associação espacial entre índice de carência social com coeficientes de detecção de casos de hanseníase 22. Do mesmo modo, em município do Maranhão, conclui-se que a condição de hiperendemicidade esteve associada à baixa escolaridade e renda 23. Dados semelhantes foram encontrados em uma pesquisa realizada na Índia, onde $71,4 \%$ dos casos abordados viviam com o equivalente a R $\$ 25,00$ por mês 24 . Destaca-se ainda, estudo, no Brasil, que demonstrou maior ocorrência da hanseníase entre contatos diagnosticados com hanseníase (coprevalentes), sugerindo a baixa escolaridade como fator de risco 25 . 
Essas e outras pesquisas reafirmam os contextos de vulnerabilidade em que a hanseníase se sustenta como problema de saúde pública, confirmando a importância de intervenções integradas com outras doenças e outros setores, como as secretarias de educação e de desenvolvimento social. Desse modo, a hanseníase mantém-se como um dos desafios no Sistema Único de Saúde (SUS) em termos da operacionalização do conceito ampliado de saúde mediante atenção integral e equânime 26. Corroborando com essas perspectivas anteriores, estudo realizado no Brasil demonstrou que o aumento da cobertura do PBF implicou na redução dos coeficientes de detecção de casos novos dessa doença 4.

Obviamente que a verificação de muitas pessoas residindo com casos de hanseníase sem tratamento remete-se a maior número de pessoas expostas cotidianamente a $M$. leprae, o que, neste estudo, traduziu em nível de significância entre sobreposição de casos e RCD com a densidade de moradores por domicílio. No entanto, por se tratar de uma situação mais comum em contextos de maior vulnerabilidade social, relacionada a condições de vida, possivelmente outros determinantes podem estar sobrepostos nesses cenários. Os resultados, mais uma vez, revelam a importância de se aprofundarem as análises a respeito do papel dos aspectos ambientais e das condições de moradia na manutenção da doença ativa em RCD. Outro estudo realizado no Brasil também demonstrou forte associação entre a média de contatos residentes e a detecção de casos novos 27,28. Domicílios com maior número de residentes e com a presença de casos de hanseníase devem ser considerados pelos serviços de saúde como espaços prioritários para as ações de vigilância, como também para construção de uma proposta de Projeto Terapêutico Singular pela atenção primária à saúde.

A ocorrência de episódios reacionais hansênicos indica a expressão de processos inflamatórios agudos ou subagudos que podem ocorrer durante toda a evolução da doença. Algumas condições predispõem o seu desenvolvimento, a exemplo de doenças intercorrentes (sobretudo infecções), gestação, puerpério, estresse físico e emocional, assim como instabilidade imunológica 29. Mediante a sua ocorrência, é essencial considerar a necessidade de uma condução próxima e qualificada do caso pela sua forte relação com progressão e piora do grau de incapacidade física, assim como pela sua possível associação com carga bacilar positiva e pelo fato de ser possível confundi-la com recidivas quando ocorrem no momento pós-alta da poliquimioterapia (PQT) 3,29,30.

A provável vinculação da reação hansênica com desfechos negativos (morte) para a pessoa acometida pela hanseníase também necessita ser considerada 31. Desse modo, a sua associação na análise multivariada com a sobreposição de casos de hanseníase em uma RCD, conforme identificado neste estudo, amplifica a complexidade da doença que, muitas vezes, está relacionada a falhas operacionais dos serviços de saúde 30. Não foram identificados outros estudos que discutam, em particular, essas duas temáticas. Portanto, o desconhecimento da magnitude do problema da reação hansênica e a sua relação com a sobreposição de casos de hanseníase em RCD fortalecem a importância de se estabelecer novos mecanismos e indicadores de vigilância para o acompanhamento desses eventos no cotidiano dos serviços de saúde 32 .

Reconhece-se como limitação do estudo a não abordagem de quase $2 / 3$ dos casos notificados por questões operacionais. Além disso, é possível a ocorrência de viés de memória, considerando tratar-se de casos notificados desde o ano de 2001. Destaca-se, no entanto, a redução de dados inconsistentes mediante a complementação de informações pela busca sistemática em prontuários e na base de dados do SINAN. Em alguns casos, não foi possível identificar variáveis importantes para o estudo nas fontes secundárias, que não tinham confirmação de informações, sobretudo clínicas, a partir dos entrevistados. A despeito dessas limitações, a abordagem de três cenários distintos do país, com diferentes graus de endemicidade, em uma casuística significativa em uma série temporal longa e com uma abordagem inovadora, reforça a importância deste estudo. Abrem-se novas possibilidades para que se avancem os estudos que integrem aspectos relativos ao controle da doença nos territórios endêmicos quando da abordagem e seguimento de contatos em RCD.

\section{Conclusão}

Reconheceu-se a elevada magnitude da ocorrência de sobreposição de casos de hanseníase em RCD em diferentes cenários de endemicidade no Brasil. O estudo revela, ainda, para além de aspectos clínico-epidemiológicos específicos determinantes, situações claras de vulnerabilidade social. Esse 
cenário demanda o enfrentamento da hanseníase por meio da incorporação de novas estratégias para efetivar a vigilância sistemática e de qualidade da rede de contatos dos casos diagnosticados, superando a abordagem exclusiva e limitada de contatos intradomiciliares avaliados.

A sobreposição de casos deve ser utilizada como um indicador sentinela de gravidade epidemiológica e operacional para a vigilância em saúde. RCD com sobreposição da doença, principalmente aquelas que enfrentam situações de vulnerabilidades (individuais, sociais e programáticas), situadas em áreas de maior endemicidade e envolvendo casos com ocorrência de episódios reacionais, devem ser consideradas prioritárias pela vigilância. Desse modo, a sobreposição de casos de hanseníase na mesma RCD é um elemento marcador crítico de distanciamento das ações da integralidade da atenção. Reforça-se a recomendação de longitudinalidade do cuidado, com seguimento por, no mínimo, cinco anos dessas pessoas, com vistas à garantia de acesso e de qualidade das ações desenvolvidas no território vivo em que se expressam as relações sociais.

\section{Colaboradores}

R. N. Boigny, E. A. Souza e A. N. Ramos Jr. contribuíram com a concepção do projeto, análise e interpretação dos dados, redação, revisão crítica relevante do conteúdo intelectual e aprovação final da versão a ser publicada. H. S. B. Romanholo, O. D. Araújo, M. A. G. Carneiro, M. D. F. Grijó e A. S. Reis contribuíram com a análise e interpretação dos dados, redação, revisão crítica relevante do conteúdo intelectual e aprovação final da versão a ser publicada. T. M. E. Araújo, N. L. F. B. Henz, M. S. A. P. Pinto e J. C. Barbosa contribuíram com a revisão crítica relevante do conteúdo intelectual e aprovação final da versão a ser publicada.

\section{Informações adicionais}

ORCID: Reagan Nzundu Boigny (0000-0002-44875509); Eliana Amorim de Souza (0000-0002-96533164); Helizandra Simoneti Bianchini Romanholo (0000-0001-9255-2635); Olivia Dias de Araújo (0000-0002-9974-4338); Telma Maria Evangelista de Araújo (0000-0001-5628-9577); Maria Angélica Gomes Carneiro (0000-0001-9548-6629); Monique Dutra Fonseca Grijó (0000-0002-6247-4952); Nubia de Lourdes Ferreira Bastos Henz (00000003-2512-7596); Adriana da Silva dos Reis (00000002-5397-1279); Maria Solange Araújo Paiva Pinto (0000-0002-3664-3927); Jaqueline Caracas Barbosa (0000-0001-6401-2244); Alberto Novaes Ramos Jr. (0000-0001-7982-1757).

\section{Agradecimentos}

Às Secretarias Municipais de Saúde dos municípios envolvidos pelas parcerias firmadas, sem as quais não seria possível o sucesso desta pesquisa. A todos os pesquisadores envolvidos durante as diferentes fases destas pesquisas, que se dedicaram dia e noite. Ao Conselho Nacional de Desenvolvimento Científico e Tecnológico (CNPq) e à organização Netherlands Hanseniasis Relief - Brasil (NHR - Brasil) pelo suporte financeiro. 


\section{Referências}

1. Freitas LRS, Duarte, EC, Garcia LP. Análise da situação epidemiológica da hanseníase em uma área endêmica no Brasil: distribuição espacial dos períodos 2001-2003 e 2010-2012. Rev Bras Epidemiol 2017; 20:702-13.

2. Departamento de Vigilância das Doenças Transmissíveis, Secretaria de Vigilância em Saúde, Ministério da Saúde. Indicadores epidemiológicos e operacionais de hanseníase. Brasil 2001-2017. http://portalms.saude.gov. br/images/pdf/2018/julho/13/Indicadoresepidemiologicos-e-operacionais-de-hansenia se-Brasil-2001-a-2017.pdf (acessado em 25/ Set/2018)

3. Departamento de Vigilância das Doenças Transmissíveis, Secretaria de Vigilância em Saúde, Ministério da Saúde. Diretrizes para vigilância, atenção e eliminação da hanseníase como problema de saúde pública. Manual técnico-operacional. http://portalms.saude.gov. br/images/pdf/2017/novembro/22/Guia-Pra tico-de-Hanseniase-WEB.pdf (acessado em 25/ Set/2018).

4. Nery JS, Pereira SM, Rasella D, Penna ML, Aquino R, Rodrigues LC, et al. Effect of the Brazilian conditional cash transfer and primary health care programs on the new case detection rate of leprosy. PLoS Negl Trop Dis 2014; 8:e3357.

5. Lopes VAS, Rangel EM. Hanseníase e vulnerabilidade social: uma análise do perfil socioeconômico de usuários em tratamento irregular. Saúde Debate 2014; 38:817-29.

6. Mieras LF, Anand S, van Brakel WH, Hamilton HC, Martin Kollmann KH, Mackenzie C, et al. Neglected Tropical Diseases, Cross-Cutting Issues Workshop, 4-6 February 2015, Utrecht, the Netherlands: meeting report. Int Health 2016; 8 Suppl 1:i7-11.

7. World Health Organization. Global Leprosy Strategy 2016-2020. Accelerating towards a leprosy-free world. http://www.searo.who. int/entity/global_leprosy_programme/docu ments/global_leprosy_strategy_2020/en/ (acessado em 25/Set/2018).

8. Ministério da Saúde. Portaria no 3.125, de 7 de outubro de 2010. Aprova as Diretrizes para Vigilância, Atenção e Controle da Hanseníase. Diário Oficial da União 2010; 15 out.

9. Romero-Montoya M, Beltran-Alzate JC, Cardona-Castro N. Evaluation and monitoring of Mycobacterium leprae transmission in household contacts of patients with Hansen's disease in Colombia. PLoS Negl Trop Dis 2017; 11:e0005325.

10. Araujo S, Freitas LO, Goulart LR, Goulart IMB. Molecular evidence for the aerial route of infection of Mycobacterium leprae and the role of asymptomatic carriers in the persistence of leprosy. Clin Infect Dis 2016; 63:1412-20.

11. Merle CS, Cunha SS, Rodrigues LC. BCG vaccination and leprosy protection: review of current evidence and status of BCG in leprosy control. Expert Rev Vaccines 2010; 9:209-22.
12. Moura MLN, Dupnik KM, Sampaio GAA Nóbrega PFC, Jeronimo AK, do NascimentoFilho JM, et al. Active surveillance of Hansen's disease (leprosy): importance for case finding among extra-domiciliary contacts. PLoS Negl Trop Dis 2013; 7:e2093.

13. Cunha EM, Giovanella L. Longitudinalidade/ continuidade do cuidado: identificando dimensões e variáveis para a avaliação da atenção primária no contexto do sistema público de saúde brasileiro. Ciênc Saúde Coletiva 2011; 16 Suppl 1:1029-42.

14. Sousa GS, Silva RLF, Xavier MB. Hanseníase e atenção primária à saúde: uma avaliação de estrutura do programa. Saúde Debate 2017; 41:230-42.

15. Durães SMB, Guedes LS, Cunha MD, Magnanini MMF, Oliveira MLWDR. Epidemiologic study of 107 cases of families with leprosy in Duque de Caxias, Rio de Janeiro, Brazil. An Bras Dermatol 2010; 85:339-45.

16. Grupo de Pesquisas Clínico-Epidemiológicas e Operacionais em Doenças Tropicais Negligenciadas. Atenção à saúde para hanseníase em áreas de alta endemicidade nos estados de Rondônia, Tocantins e Bahia: abordagem integrada de aspectos operacionais, epidemiológicos (espaço-temporais), clínicos e psicossociais (Integrahans-Norte e Nordeste). Relatório do projeto. Fortaleza: Universidade Federal do Ceará; 2017.

17. Deps PD, Guedes BVS, Bucker Filho J, Andreatta MK, Marcari RS, Rodrigues LC. Characteristics of known leprosy contact in a high endemic area in Brazil. Lepr Rev 2006; 77:34-40.

18. Bakker MI, Hatta M, Kwenang A, Faber WR van Beers SM, Klaster PR, et al. Population survey to determine risk factors for Mycobacterium leprae transmission and infection. Int $\mathrm{J}$ Epidemiol 2004; 33:1329-36.

19. Amorim FM, Nobre ML, Ferreira LC, Nascimento LS, Miranda AM, Monteiro GR, et al. Identifying leprosy and those at risk of developing leprosy by detection of antibodies against LID-1 and LID-NDO. PLoS Negl Trop Dis 2016; 10:e0004934.

20. Ayres JRCM, França Júnior I, Calazans GJ, Saletti Filho HC, Czeresnia D, Freitas CM. O conceito de vulnerabilidade e as práticas de saúde: novas perspectivas e desafios. In: Czeresina D, Freita CM, organizadores. Promoção da saúde: conceitos, reflexões, tendências. Rio de Janeiro: Editora Fiocruz; 2003. p. 117-39.

21. Imbiriba ENB, Silva Neto AL, Souza WV, Pedrosa V, Cunha MG, Garnelo L. Social inequality, urban growth and leprosy in Manaus: a spatial approach. Rev Saúde Pública 2009; 43:656-65

22. Chaves EC, Costa SV, Flores RLR, Neves EOS. Índice de carência social e hanseníase no Estado do Pará em 2013: análise espacial. Epidemiol Serv Saúde 2017; 26:807-16. 
23. Aquino DMC, Caldas AJM, Silva AAM, Costa JML. Perfil dos pacientes com hanseníase em área hiperendêmica da Amazônia do Maranhão, Brasil. Rev Soc Bras Med Trop 2003; 36:57-64.

24. Nair SP. Leprosy in families: clinicoepidemiological profile from a tertiary care centre. Indian Dermatol Online J 2017; 8:328-30.

25. Sales AM, Ponce de Leon A, Düppre NC, Hacker MA, Nery JAC, Sarno EN, et al. Leprosy among patient contacts: a multilevel study of risk factors. PLoS Negl Trop Dis 2011; 5:e1013.

26. Mattos RA. A integralidade na prática (ou sobre a prática da integralidade). Cad Saúde Pública 2004; 20:1411-6.

27. Brito MFM, Ximenes RAA, Gallo MEN, Bührer-Sékula S. Association between leprosy reactions after treatment and bacterial load evaluated using anti PGL-I serology and bacilloscopy. Rev Soc Bras Med Trop 2008; 41:6772.

28. Castro SS, Santos JPP, Abreu GB, Oliveira VR, Fernandes LFRM. Leprosy incidence, characterization of cases and correlation with household and cases variables of the Brazilian states in 2010. An Bras Dermatol 2016; 91:28-33.
29. Teixeira MAG, Silveira VM, França ER. Características epidemiológicas e clínicas das reações hansênicas em indivíduos paucibacilares e multibacilares, atendidos em dois centros de referência para hanseníase, na cidade de Recife, Estado de Pernambuco. Rev Soc Bras Med Trop 2010; 43:287-92.

30. Souza EA, Boigny RN, Ferreira AF, Alencar $\mathrm{CH}$, Oliveira MLW, Ramos Jr. AN. Vulnerabilidade programática no controle da hanseníase: padrões na perspectiva de gênero no Estado da Bahia, Brasil. Cad Saúde Pública 2018; 34:e00196216.

31. Souza EA, Boigny RN, Oliveira HX, Oliveira MLW, Heukelbach J, Alencar CH, et al. Tendências e padrões espaço-temporais da mortalidade relacionada à hanseníase no Estado da Bahia, Nordeste do Brasil, 1999 a 2014. Cad Saúde Colet (Rio J.) 2018; 26:191-202.

32. Steinmann P, Reed SG, Mirza F, Hollingsworth TD, Richardus JH. Innovative tools and approaches to end the transmission of Mycobacterium leprae. Lancet Infect Dis 2017; 17:e298e305. 


\section{Abstract}

The study sought to analyze the magnitude of occurrence and the sociodemographic, economic and clinical profiles of leprosy associated with household social networks (HSN), with disease overlap in cities from the states of Bahia, Piaui and Rondônia, Brazil, from 2001 to 2014. This is a cross-sectional study using primary and secondary data regarding new cases of leprosy notified to the Brazilian Information System for Notifiable Diseases (SINAN, in Portuguese) residing in the cities. We applied a standardized instrument to the new cases and reviewed data from charts and from SINAN. Of a total of 1,032 (29.6\%) assessed cases, 538 (52.1\%) had more than one case in their $H S N$. There were larger frequencies of female sex (292; 54.3\%), age between 41 and 60 years (240; $44.6 \%)$, primary education (272; 50.6\%), income lower than the minimum wage $(265 ; 49.3 \%)$ and living with 5 or more people $(265 ; 49.3 \%)$. The overlap of cases in the HSN was associated in the multivariate analysis with residing in cities in the state of Rondônia ( $P R=1.23$; 95\%CI: 1.07-1.43; $p=0.003)$, as well as living with 3 to 4 people in the same household ( $P R=1.66$; 95\%CI: 1.112.49; $p=0.014)$ and having leprosy reaction $(P R$ $=1.31$; 95\% CI: 0.99-1.70; $p=0.050$ ). Case repetition within the same HSN is a frequent event in the situations we studied. Its occurrence must be considered as a sentinel indicator of greater epidemiological severity in primary health care surveillance. We highlight the vulnerability of affected families.

Leprosy; Infectious Disease Transmission; Epidemiological Monitoring

\section{Resumen}

El objetivo de este estudio fue analizar la magnitud de la ocurrencia y los perfiles sociodemográficos, económicos y clínicos de casos de lepra, vinculados a las redes de convivencia domiciliaria $(R C D)$, con sobreposición de la enfermedad, en municipios de los estados de Bahía, Piauí y Rondônia, Brasil, durante el período de 2001 a 2014. Se trata de un estudio transversal, con datos primarios y secundarios de casos nuevos de lepra, notificados en el Sistema de Información de Enfermedades de Obligada Notificación (SINAN, por su sigla en portugués) y residentes en los municipios. Se procedió a la aplicación de un instrumento estandarizado a los casos nuevos y a la revisión de datos en prontuarios y base de datos del SINAN. De un total de $1.032(29,6 \%)$ casos de lepra abordados, $538(52,1 \%)$ tenían más de un caso en su $R C D$. Existía una mayor frecuencia de personas del sexo femenino (292; 54,3\%), con edades comprendidas entre 41 y 60 años (240; 44,6\%), enseñanza fundamental (272; 50,6\%), renta menor a un salario mínimo $(265 ; 49,3 \%)$ y residiendo con 5 personas o más (265; 49,3\%). La ocurrencia de sobreposición de casos en la $R C D$ se asoció en el análisis multivariado a residir en municipios del estado de Rondônia ( $R P=1,23$; IC95\%: 1,07-1,43; $p=0,003)$, así como vivir con de 3 a 4 personas en el mismo domicilio ( $R P=1,66$; IC95\%: 1,112,49; $p=0,014)$ y sufrir reacción leprótica $(R P=$ 1,31; IC95\%: 0,99-1,70; $p=0,050)$. La repetición de casos de lepra en una misma $R C D$ representa un evento frecuente en los escenarios abordados. Su ocurrencia debe ser considerada como un indicador centinela de mayor gravedad epidemiológica para la vigilancia en la red de atención básica en la salud. Se resalta el carácter de vulnerabilidad de las familias participantes.

Lepra; Transmisión de Enfermedad Infecciosa; Monitoreo Epidemiológico
Recebido em 30/Mai/2018

Versão final reapresentada em 26/Out/2018 Aprovado em 05/Nov/2018 